\title{
Divergence of Fourier series: Corrigenda. II
}

\section{Masako Izumi and Shin-ichi Izumi}

\begin{abstract}
The proof of divergence of Fourier series which was constructed in the authors' earlier paper [Bull. Austral. Math. Soc. 8 (1973), 289-304] is not complete. The object of this paper is to show that the unestimated terms do not disturb the divergence.
\end{abstract}

\section{Introduction}

Dr Y.M. Chen has kindly pointed out to us that the formula of $s_{m_{j}+N}\left(t ; f_{n}\right)$ in $[1], p .291$, is not complete. Besides $P_{1}, P_{2}$, and $P_{3}$, $s_{m_{j}+N}\left(t ; f_{n}\right)$ contains the terms $P_{4}$ and $P_{5}$ which will be written up as follows: $P_{4}$ and $P_{5}$ are the sums of terms of order $\leq m_{j}+N$ in

$$
P_{4}^{\prime}=\frac{1}{n} \sum_{i=j+1}^{n} \sum_{k=m_{j}^{+1}}^{m_{j}^{+N}}\left(1-\frac{k}{m_{i}+1}\right) \cos k\left(t-c_{i}\right) \cdot\left(s_{N}(t ; h)-s_{N+m_{j}} k^{(t ; h)}\right)
$$

and

$$
P_{5}^{\prime}=\frac{1}{n} \sum_{i=j+1}^{n} \sum_{k=m_{j}+N+1}^{m_{j}^{+}+2 N}\left(1-\frac{k}{m_{i}+1}\right) \cos k\left(t-c_{i}\right) s_{! N}(t ; h),
$$

respectively.

We shall estimate $P_{4}$ and $P_{5}$ and prove that the proposition stated in Section 2 of [1] is still true.

Received 14 November 1973. 


\section{Estimation of $P_{4}$}

Writing

$$
s_{N}(t ; h)=\sum_{l=0}^{N}\left(\alpha_{2} \cos Z t+\beta_{\eta} \sin Z t\right),
$$

the sum of terms of order $\leq m_{j}+N$ in the term

$$
\cos k\left(t-c_{i}\right)\left(s_{N}(t ; h)-s_{N+m_{j}-k}(t ; h)\right)
$$

of $P_{4}^{\prime}$, is

$$
\begin{aligned}
& \frac{1}{2} \sum_{Z=N+m_{j}-k+1}^{N}\left\{\alpha_{2} \cos \left((k-Z) t-k c_{i}\right)-B_{2} \sin \left((k-l) t-k c_{i}\right)\right\} \\
& =\frac{1}{2 \pi} \sum_{\imath=N+m_{j}-k+1}^{N} \int_{0}^{2 \pi} h(u)\left\{\cos 2 u \cos \left((k-l) t-k c_{i}\right)-\sin Z u \sin \left((k-Z) t-k c_{i}\right)\right\} d u \\
& =\frac{1}{2 \pi} \sum_{\imath=N+m_{j}-k+1}^{N} \int_{0}^{2 \pi} h(u) \cos \left(Z(u-t)+k\left(t-c_{i}\right)\right) d u
\end{aligned}
$$

Therefore

$$
\begin{aligned}
& P_{4}= \frac{1}{2 \pi n} \sum_{i=j+1}^{n} \sum_{k=m_{j}^{+1}}^{m_{j}^{+N}}\left(1-\frac{k}{m_{i}+1}\right) \sum_{z=N+m_{j}-k+1}^{N} \int_{0}^{2 \pi} h(u) \cos \left(2(u-t)+k\left(t-c_{i}\right)\right) d u \\
&= \frac{1}{2 \pi n} \sum_{i=j+1}^{n} \sum_{k=m_{j}^{+1}}^{m_{j}^{+N}}\left(1-\frac{k}{m_{i}+1}\right) \int_{0}^{2 \pi} \frac{h(u)}{2 \sin (u-t) / 2} \\
& \quad \cdot\left\{\sin \left((N+1 / 2)(u-t)+k\left(t-c_{i}\right)\right)-\sin \left(\left(N+m_{j}-k+1 / 2\right)(u-t)+k\left(t-c_{i}\right)\right)\right\} d u \\
&=P_{41}-P_{42},
\end{aligned}
$$

where 


$$
\begin{aligned}
P_{41}= & \frac{1}{2 \pi n} \sum_{i=j+1}^{n} \sum_{k=m_{j}+1}^{m_{j}+N} \int_{0}^{2 \pi} \frac{h(u)}{2 \sin (u-t) / 2} \\
& \cdot\left\{\sin \left((N+1 / 2)(u-t)+k\left(t-c_{i}\right)\right)-\sin \left(\left(N+m_{j}-k+1 / 2\right)(u-t)+k\left(t-c_{i}\right)\right)\right\} d u \\
= & \frac{1}{2 \pi n} \sum_{i=j+1}^{n} \int_{0}^{2 \pi} \frac{h(u)}{2 \sin (u-t) / 2} \cdot\left\{\left[\cos \left((N+1 / 2)(u-t)+\left(m_{j}+1 / 2\right)\left(t-c_{i}\right)\right\}\right.\right. \\
& \left.-\cos \left((N+1 / 2)(u-t)+\left(m_{j}+N+1 / 2\right)\left(t-c_{i}\right)\right)\right] \frac{1}{2 \sin \left(t-c_{i}\right) / 2} \\
& \left.-\left[\cos \left(N(u-t)+\left(m_{j}+1 / 2\right)\left(t-c_{i}\right)\right)-\cos \left(m_{j}+N+1 / 2\right)\left(t-c_{i}\right)\right] \frac{1}{2 \sin \left(2 t-u-c_{i}\right) / 2}\right\} d u .
\end{aligned}
$$

Let $n_{0}$ be the minimum of $n$ and $[(2 n+1) t / 2]$ and $I_{i}$ be the interval $\left(c_{k}, c_{k+1}\right)$ containing the point $2 t-c_{i}$, then

$$
\begin{aligned}
P_{41}=\frac{1}{2 \pi n} & \sum_{i=j+1}^{n_{0}} \int_{I_{i}} \frac{h(u)}{2 \sin (u-t) / 2} \cdot\left\{\left[\cos \left((N+1 / 2)(u-t)+\left(m_{j}+1 / 2\right)\left(t-c_{i}\right)\right)\right.\right. \\
& \left.-\cos \left((N+1 / 2)(u-t)+\left(m_{j}+N+1 / 2\right)\left(t-c_{i}\right)\right)\right] \frac{1}{2 \sin \left(t-c_{i}\right) / 2} \\
& \left.-\left[\cos \left(N(u-t)+\left(m_{j}+1 / 2\right)\left(t-c_{i}\right)-\cos \left(m_{j}+N+1 / 2\right)\left(t-c_{i}\right)\right)\right] \frac{1}{2 \sin \left(2 t-u-c_{i}\right) / 2}\right\} d u \\
& +\frac{1}{2 \pi n} \sum_{i=j+1}^{n} \int_{c_{j}}^{c_{j+1}} \frac{h(u)}{2 \sin (u-t) / 2} \\
& -\left\{\left[\cos \left((N+1 / 2)(u-t)+\left(m_{j}+1 / 2\right)\left(t-c_{i}\right)\right) \sin \left(2 t-u-c_{i}\right) / 2\right.\right. \\
& \left.-\cos \left(N(u-t)+\left(m_{j}+1 / 2\right)\left(t-c_{i}\right)\right) \sin \left(t-c_{i}\right) / 2\right] \\
& -\left[\cos \left((N+1 / 2)(u-t)+\left(m_{j}+N+1 / 2\right)\left(t-c_{i}\right)\right) \sin \left(2 t-u-c_{i}\right) / 2\right. \\
& \left.\left.-\cos \left(m_{j}+N+1 / 2\right)\left(t-c_{i}\right) \sin \left(t-c_{i}\right) / 2\right]\right\} \\
& +\frac{1}{2 \sin \left(t-c_{i}\right) / 2 \sin \left(2 t-u-c_{i}\right) / 2} d u+O(1) \\
=Q_{41} & +Q_{42}+0(1) \cdot
\end{aligned}
$$

Since

$$
\begin{aligned}
\cos \left((N+1 / 2)(u-t)+\left(m_{j}+1 / 2\right)\left(t-c_{i}\right)\right)-\cos \left((N+1 / 2)(u-t)+\left(n_{j}+N+1 / 2\right)\left(t-c_{i}\right)\right) \\
=2 \operatorname{sinil}\left(t-c_{i}\right) / 2 \cdot \sin \left((i l+1 / 2)(u-t)+\left(m_{j}+(j+1) / 2\right)\left(t-c_{i}\right)\right)
\end{aligned}
$$


and

$$
\begin{aligned}
\cos \left(N(u-t)+\left(m_{j}+1 / 2\right)\right. & \left.\left(t-c_{i}\right)\right)-\cos \left(m_{j}+N+1 / 2\right)\left(t-c_{i}\right) \\
= & \cos \left(N\left(u-\left(2 t-c_{i}\right)\right)+\left(m_{j}+N+1 / 2\right)\left(t-c_{i}\right)\right)-\cos \left(m_{j}+N+1 / 2\right)\left(t-c_{i}\right) \\
= & \left(\cos N\left(u-\left(2 t-c_{i}\right)\right)-1\right) \cos \left(m_{j}+N+1 / 2\right)\left(t-c_{i}\right) \\
& \quad-\sin \left(m_{j}+N+1 / 2\right)\left(t-c_{i}\right) \sin N\left(u-\left(2 t-c_{i}\right)\right),
\end{aligned}
$$

we get

$$
\begin{aligned}
& Q_{41}=\frac{1}{2 \pi n} \sum_{i=j+1}^{n_{0}} \int_{I_{i}} \frac{h(u)}{2 \sin (u-t) / 2} \\
& \text { - }\left\{\frac{\cos N\left(u-\left(2 t-c_{i}\right)\right)-1}{2 \sin \left(2 t-u-c_{i}\right) / 2} \cos \left(m_{j}+N+1 / 2\right)\left(t-c_{i}\right)\right. \\
& \left.-\frac{\sin N\left(u-\left(2 t-c_{i}\right)\right)}{2 \sin \left(2 t-u-c_{i}\right) / 2} \sin \left(m_{j}+N+1 / 2\right)\left(t-c_{i}\right)\right\} d u+o(1) \\
& =\frac{1}{2 n} \sum_{i=j+1}^{n} \frac{1}{2 \sin \left(t-c_{i}\right) / 2} \cdot\left(\cos \left(m_{j}+N+1 / 2\right)\left(t-c_{i}\right) \bar{s}_{N}\left(2 t-c_{i} ; h\right)\right. \\
& \left.+\sin \left(m_{j}+i v+1 / 2\right)\left(t-c_{i}\right) s_{i j}\left(2 t-c_{i} ; h\right)\right\} d u+O(1) \\
& =\frac{1}{2 n}\left\{\cos \left(m_{j}+N+1 / 2\right) t \cdot \bar{s}_{N}\left(2 t-c_{i} ; \eta\right)+\sin \left(m_{j}+N+1 / 2\right) t \cdot s_{N}\left(2 t-c_{i} ; h\right)\right\} \\
& \sum_{i=j+1}^{n_{0}} \frac{1}{2 \sin \left(t-c_{i}\right) / 2}+o(1) \\
& =\frac{1}{2 n}\left\{\cos \left(m_{j}+N+1 / 2\right) t \cdot \bar{h}\left(2 t-c_{j}\right)+\sin \left(m_{j}+N+1 / 2\right) t\right\} \\
& \text { - } \sum_{i=j+1}^{n_{0}} \frac{1}{2 \sin \left(t-c_{i}\right) / 2}+O(1)
\end{aligned}
$$

since $h\left(2 t-c_{j}\right)=1$.

We shall now estimate $Q_{42}$. Since

$$
\begin{aligned}
& \cos \left((N+1 / 2)(u-t)+\left(m_{j}+1 / 2\right)\left(t-c_{i}\right)\right) \sin \left(2 t-u-c_{i}\right) / 2 \\
&-\cos \left(l l(u-t)+\left(m_{j}+1 / 2\right)\left(t-c_{i}\right)\right) \sin \left(t-c_{i}\right) / 2 \\
&=-\cos \left((I J+1 / 2)(u-t)+\left(m_{j}+1\right)\left(t-c_{i}\right)\right) \sin (u-t) / 2
\end{aligned}
$$


and

$$
\begin{aligned}
& \cos ((N+1 / 2)(u-t)+\left.\left(m_{j}+N+1 / 2\right)\left(t-c_{i}\right)\right) \sin \left(2 t-u-c_{i}\right) / 2 \\
& \quad-\cos \left(m_{j}+N+1 / 2\right)\left(t-c_{i}\right) \sin \left(t-c_{i}\right) / 2 \\
&=(\cos N(u-t)-1) \cos \left(m_{j}+N+1 / 2\right)\left(t-c_{i}\right) \sin \left(t-c_{i}\right) / 2 \\
&-\sin \left(m_{j}+N+1 / 2\right)\left(t-c_{i}\right) \sin (N+1 / 2)(u-t) \cos (t-u) / 2 \sin \left(t-c_{i}\right) / 2 \\
&-\cos \left(m_{j}+N+1 / 2\right)\left(t-c_{i}\right) \cos (N+1 / 2)(u-t) \sin (t-u) / 2 \cos \left(t-c_{i}\right) / 2 \\
& \quad+\sin (N+1 / 2)(u-t) \sin (u-t) / 2 \sin \left(m_{j}+N\right)\left(t-c_{i}\right),
\end{aligned}
$$

we have

$$
\begin{aligned}
Q_{42}= & \frac{-1}{2 \pi n} \sum_{i=j+1}^{n} \int_{c_{j}}^{c} j+1 \frac{h(u)}{2 \sin \left(2 t-u-c_{i}\right) / 2} \cdot\left\{\cos \left(m_{j}+N+1 / 2\right)\left(t-c_{i}\right) \frac{\cos N(u-t)-1}{2 \sin (u-t) / 2}\right. \\
=\frac{1}{2 n} \sum_{i=j+1}^{n} \frac{1}{2 \sin \left(t-c_{i}\right) / 2} & \left.-\sin \left(m_{j}+N+1 / 2\right)\left(t-c_{i}\right) \frac{\sin (N+1 / 2)(u-t)}{2 \sin (u-t) / 2}\right\} d u+o(1) \\
& \cdot\left\{\cos \left(m_{j}+N+1 / 2\right)\left(t-c_{i}\right) \bar{s}_{N}(t ; h)+\sin \left(m_{j}+N+1 / 2\right)\left(t-c_{i}\right) s_{N}(t ; h)\right\}+O(1) \\
=\frac{1}{2 n} & \left\{\cos \left(m_{j}+N+1 / 2\right) t \cdot \bar{h}(t)+\sin \left(m_{j}+N+1 / 2\right) t\right\} \cdot \sum_{i=j+1}^{n} \frac{1}{2 \sin \left(t-c_{i}\right) / 2}+O(1) .
\end{aligned}
$$

\section{Therefore}

$$
\begin{aligned}
P_{41}= & \frac{1}{2 n}\left\{\cos \left(m_{j}+N+1 / 2\right) t \cdot \bar{h}\left(2 t-c_{i}\right)+\sin \left(m_{j}+N+1 / 2\right) t\right\} \cdot \sum_{i=j+1}^{n_{0}} \frac{1}{2 \sin \left(t-c_{i}\right) / 2} \\
& +\frac{1}{2 n}\left\{\cos \left(m_{j}+N+1 / 2\right) t \cdot \bar{h}(t)+\sin \left(m_{j}+N+1 / 2\right) t\right\} \cdot \sum_{i=j+1}^{n} \frac{1}{2 \sin \left(t-c_{i}\right) / 2}+o(1) .
\end{aligned}
$$

By similar extimation, we can see that

$$
P_{42}=O\left((\log \log n)^{2}\right) \text {. }
$$

\section{Estimation of $P_{5}$}

The sum of terms of order $\leq m_{j}+W$ in

$$
\cos k\left(t-c_{i}\right) s_{N}(t ; h)
$$


of $P_{5}^{\prime}$, is

$$
\begin{aligned}
& \frac{1}{2} \sum_{Z=k-m_{j}-N}^{N}\left\{\alpha_{i} \cos \left((k-Z) t-k c_{i}\right)-B_{Z} \sin \left((k-Z) t-k c_{i}\right)\right\} \\
& =\frac{1}{2 \pi} \sum_{l=k-m_{j}-l l}^{N} \int_{0}^{2 \pi} h(u)\left\{\cos \left((k-l) t-k c_{i}\right) \cos Z u-\sin \left((k-l) t-k c_{i}\right) \sin Z u\right\} d u \\
& =\frac{1}{2 \pi} \sum_{l=k-m_{j}-N}^{N} \int_{0}^{2 \pi} h(u) \cos \left(z(u-t)+k\left(t-c_{i}\right)\right) d u \text {; }
\end{aligned}
$$

and then

$$
\begin{aligned}
P_{5}= & \frac{1}{2 \pi n} \sum_{i=j+1}^{n} \sum_{k=m_{j}^{+N+1}}^{m_{j}^{+2 N}}\left(1-\frac{k}{m_{i}+1}\right) \cdot \sum_{i=k-m_{j}-N}^{N} \int_{0}^{2 \pi} h(u) \cos \left(z(u-t)+k\left(t-c_{i}\right)\right) d u \\
= & \frac{1}{2 \pi n} \sum_{i=j+1}^{n} \sum_{k=m_{j}^{+N+1}}^{m_{j}^{+2 N}}\left(1-\frac{k}{m_{i}+1}\right) \int_{0}^{2 \pi} \frac{h(u)}{2 \sin (u-t) / 2} \\
& \cdot\left\{\sin \left((N+1 / 2)(u-t)+k\left(t-c_{i}\right)\right)-\sin \left(\left(k-m_{j}-N-1 / 2\right)(u-t)+k\left(t-c_{i}\right)\right)\right\} d u \\
= & P_{51}-P_{52},
\end{aligned}
$$

where 


$$
\begin{aligned}
& P_{51}=\frac{1}{2 \pi n} \sum_{i=j+1}^{n} \sum_{k=m_{j}+N+1}^{m+2 N} \int_{0}^{2 \pi} \frac{h(u)}{2 \sin (u-t) / 2} \\
& \text { - }\left\{\sin \left((N+1 / 2)(u-t)+k\left(t-c_{i}\right)\right)-\sin \left(\left(k-m_{j}-N-1 / 2\right)(u-t)+k\left(t-c_{i}\right)\right)\right\} d u \\
& =\frac{1}{2 \pi n} \sum_{i=j+1}^{n} \int_{0}^{2 \pi} \frac{h(u)}{2 \sin (u-t) / 2} \\
& \text { - }\left\{\left[\cos \left((N+1 / 2)(u-t)+\left(m_{j}+i+1 / 2\right)\left(t-c_{i}\right)\right)\right.\right. \\
& \left.-\cos \left((N+1 / 2)(u-t)+\left(m_{j}+2 N+1 / 2\right)\left(t-c_{i}\right)\right)\right] \frac{1}{2 \sin \left(t-c_{i}\right) / 2} \\
& \left.-\left[\cos \left(m_{j}+N+1 / 2\right)\left(t-c_{i}\right)-\cos \left(u(u-t)+\left(m_{j}+2 N+1 / 2\right)\left(t-c_{i}\right)\right)\right] \frac{1}{2 \sin \left(u-c_{i}\right) / 2}\right\} d u \\
& =\frac{1}{2 \pi n} \sum_{i=j+1}^{n} \int_{0}^{2 \pi} \frac{h(u)}{2 \sin (u-t) / 2} \\
& \text { - }\left\{[\cos (N+1 / 2)(u-t)-\cos (u-t) / 2] \cos \left(m_{j}+N+1 / 2\right)\left(t-c_{i}\right) \sin \left(u-c_{i}\right) / 2\right. \\
& -\sin (N+1 / 2)(u-t) \sin \left(m_{j}+N+1 / 2\right)\left(t-c_{i}\right) \sin \left(t-c_{i}\right) / 2 \\
& -\cos \left(m_{j}+N+1 / 2\right)\left(t-c_{i}\right) \cos \left(u-c_{i}\right) / 2 \sin (t-u) / 2 \\
& \left.-\cos \left((N+1 / 2)(u-t)+\left(m_{j}+2 N+1\right)\left(t-c_{i}\right)\right) \sin (u-t) / 2\right\} \\
& \frac{d u}{2 \sin \left(t-c_{i}\right) / 2 \cdot \sin \left(u-c_{i}\right) / 2} \\
& =\frac{1}{2 n} \sum_{i=j+1}^{n}\left(\cos \left(m_{j}+N+1 / 2\right)\left(t-c_{i}\right) \bar{s}_{N}(t ; h)\right. \\
& \left.-\sin \left(m_{j}+N+1 / 2\right)\left(t-c_{i}\right) s_{N}(t ; h)\right\} \frac{1}{2 \sin \left(t-c_{i}\right) / 2}+o(1) \\
& =\frac{1}{2 n}\left\{\cos \left(m_{j}+N+1 / 2\right) t \cdot \bar{h}(t)-\sin \left(m_{j}+N+1 / 2\right) t\right\} \sum_{i=j+1}^{n} \frac{1}{2 \sin \left(t-c_{i}\right) / 2}+O(1) .
\end{aligned}
$$

Similarly we can prove that

$$
P_{52}=O\left((\log \log n)^{2}\right) .
$$

4.

In [l], p. 294, the $Z$-th Fourier coefficient of $J(v)$ must be $1 / \pi z$ instead of $1 / 2 \pi l$ (see [2]). Therefore the formula in [1], p. 300, must be changed as tollows: 


$$
\begin{aligned}
P_{1}+P_{2}+ & P_{3}=\frac{1}{2 n}\left\{3 \sin \left(m_{j}+/ N+1 / 2\right) t-\sin \left(m_{j}+1 / 2\right) t\right. \\
& \left.-18 \sin \left(m_{j}+/ N+1 / 2\right) t / \log \log n\right\} \sum_{i=j+1}^{n} \frac{1}{2 \sin \left(t-c_{i}\right) / 2}+o\left((\log \log n)^{2}\right) .
\end{aligned}
$$

By the estimations in Sections 2 and 3 ,

$$
\begin{aligned}
P_{4}+P_{5}=\frac{1}{2 n} & \left\{\sin \left(m_{j}+h+1 / 2\right) t+\cos \left(m_{j}+N+1 / 2\right) t \cdot \bar{h}\left(2 t-c_{j}\right)\right\} \cdot \sum_{i=j+1}^{n_{0}} \frac{1}{2 \sin \left(t-c_{i}\right) / 2} \\
& +\frac{1}{n} \cos \left(m_{j}+N+1 / 2\right) t \cdot \bar{h}(t) \sum_{i=j+1}^{n} \frac{1}{2 \sin \left(t-c_{i}\right) / 2}+o\left((\log \log n)^{2}\right) .
\end{aligned}
$$

Therefore, if we suppose $j>n / \log n$, then

$$
\begin{aligned}
& s_{m_{j}+l}\left(t ; f_{n}\right)=P_{1}+P_{2}+P_{3}+P_{4}+P_{5} \\
& =\frac{1}{2 n}\left[\left\{3 \sin \left(m_{j}+N+1 / 2\right) t-\sin \left(m_{j}+1 / 2\right) t+2 \cos \left(m_{j}+N+1 / 2\right) t \cdot \bar{h}(t)\right.\right. \\
& \left.-18 \sin \left(m_{j}+N+1 / 2\right) t / \log \log n\right\} \sum_{i=j+1}^{n} \frac{1}{2 \sin \left(t-c_{i}\right) / 2} \\
& +\left\{\sin \left(m_{j}+N+1 / 2\right) t+\cos \left(m_{j}+N+1 / 2\right) t \cdot \bar{h}\left(2 t-c_{i}\right)\right\} \\
& \text { - } \left.\sum_{i=j+1}^{n_{0}} \frac{1}{2 \sin \left(t-c_{i}\right) / 2}\right]+o\left((\log \log n)^{2}\right) \\
& =\frac{1}{2 n}\left\{4 \sin \left(m_{j}+N+1 / 2\right) t-\sin \left(m_{j}+1 / 2\right) t\right. \\
& +2 \cos \left(m_{j}+N+1 / 2\right) t \cdot \bar{h}(t)+\cos \left(m_{j}+N+1 / 2\right) t \cdot \bar{h}\left(2 t-c_{j}\right) \\
& \left.-18 \sin \left(m_{j}+N+1 / 2\right) t / \log \log n\right) \cdot \sum_{i=j+1}^{n_{0}} \frac{1}{2 \sin \left(t-c_{i}\right) / 2} \\
& +o\left((\log \log n)^{2}\right) \\
& =\frac{1}{2 n} K(t) \sum_{i=j+1}^{n_{0}} \frac{1}{2 \sin \left(t-c_{i}\right) / 2}+O\left((\log \log n)^{2}\right) \text {. }
\end{aligned}
$$

Let $E_{j n}$ be the set of $t$ satisfying the conditions (1) and (2) in [1] and such that

$$
|K(t)|>1 / \log \log n
$$


If we put

$$
E_{n}=\bigvee\left(E_{j n} ; n / \log n<j<n-\sqrt{n}\right) \text {, }
$$

then $E_{n}$ and $f_{n}$ satisfy the conditions of the proposition stated at the beginning of Section 2 of [1].

Section 3 of [1] remains unchanged.

\section{References}

[1] Masako Izumi and Shin-ichi Izumi, "Divergence of Fourier series", Bulz. Austral. Math. Soc. 8 (1973), 289-304.

[2] Masako Izur, and Shin-ichi Izumi, "Divergence of Fourier series: Corrigenda", Bul2. Austral. Math. Soc. 9 (1973), 319-320.

Department of Mathematics, Institute of Advanced Studies, Australian National University, Canberra, ACT. 